\title{
Meditation and Cognitive Ageing: the Role of Mindfulness Meditation in Building Cognitive Reserve
}

\author{
Peter Malinowski $^{1}$ (D) Liliana Shalamanova ${ }^{2}$ \\ Received: 10 December 2016/Accepted: 5 April 2017 /Published online: 12 April 2017 \\ (C) The Author(s) 2017. This article is an open access publication
}

\begin{abstract}
Mindfulness-related meditation practices engage various cognitive skills including the ability to focus and sustain attention, which in itself requires several interacting attentional subfunctions. There is increasing behavioural and neuroscientific evidence that mindfulness meditation improves these functions and associated neural processes. More so than other cognitive training programmes, the effects of meditation appear to generalise to other cognitive tasks, thus demonstrating far transfer effects. As these attentional functions have been linked to age-related cognitive decline, there is growing interest in the question whether meditation can slowdown or even prevent such decline. The cognitive reserve hypothesis builds on evidence that various lifestyle factors can lead to better cognitive performance in older age than would be predicted by the existing degree of brain pathology. We argue that mindfulness meditation, as a combination of brain network and brain state training, may increase cognitive reserve capacity and may mitigate age-related declines in cognitive functions. We consider available direct and indirect evidence from the perspective of cognitive reserve theory. The limited available evidence suggests that MM may enhance cognitive reserve capacity directly through the repeated activation of attentional functions and of the multiple demand system and indirectly through the improvement of physiological mechanisms associated with stress and immune function. The article
\end{abstract}

Peter Malinowski

p.malinowski@ljmu.ac.uk

1 Research Centre for Brain and Behaviour, Liverpool John Moores University, Liverpool L3 3AF, UK

2 Centre for Biomedicine, School of Healthcare Science, Manchester Metropolitan University, Manchester M1 5GD, UK concludes with outlining research strategies for addressing underlying empirical questions in more substantial ways.

Keywords Mindfulness meditation · Cognitive enhancement $\cdot$ Cognitive reserve $\cdot$ Cognitive ageing $\cdot$ Far transfer $\cdot$ Brain network training $\cdot$ Brain state training

\section{Introduction}

As we grow older, many of us are likely to experience a decline in cognitive functioning, including deficits in executive control, processing speed, working memory and verbal and visuospatial memory, collectively referred to as cognitive ageing. As cognition is fundamental to health and well-being, these impairments are associated with a loss of quality of life, increased disability and higher health-related expenditures (Albert et al. 2002; DeCarli 2003; Ernst and Hay 1997; Lyketsos et al. 2002; Salthouse 2004; Tabert et al. 2002). Encouragingly, research over the past two decades suggests that the adult brain has much greater capacity for plasticity than previously thought. It has been shown that the structure of the brain adapts with experience and can change in response to training (Colcombe et al. 2006; Draganski et al. 2004; Driemeyer et al. 2008; Gage 2002). Furthermore, it appears that cognitive decline may be influenced by nonbiological factors such as education, diet, physical exercise and other life style choices (Hedden and Gabrieli 2004), thus opening the doors for preventative approaches that enable healthy brain ageing by preserving or even restoring cognitive functions.

Recently, it has been proposed that mindfulness meditation may be a useful approach for preventing age-related cognitive decline (e.g. Gard et al. 2014; Luders 2014; Malinowski et al. 2017). Mindfulness-related meditation practices have grown 
in popularity, in particular because programmes that integrate such meditation exercises showed early promise of efficacy. For instance, mindfulness-based interventions demonstrated benefits for the treatment of anxiety disorders and depression (Hofmann et al. 2010; Piet and Hougaard 2011), chronic pain (Veehof et al. 2011) and substance-use disorders (Chiesa and Serretti 2014), as well as improvements in well-being and behavioural regulation (Keng et al. 2011).

It should be noted though, that despite these promising findings, robust efficacy evidence is still limited even for the best researched mindfulness-based interventions such as mindfulness-based stress reduction (MBSR) or mindfulnessbased cognitive therapy (MBCT). At present, efficacy claims are based on relatively small numbers of studies, which do not always employ the strongest study designs. Furthermore, these interventions often do not yield better outcomes than other active treatments (Goyal et al. 2014). They may thus not be superior to established treatment approaches but rather offer a valuable alternative. Furthermore, the question whether mindfulness meditation is the central active component within these multi-component programmes has not been rigorously addressed (Malinowski 2017; Malinowski et al. 2017). A recent study on depressive relapse prevention suggests that this may not necessarily be the case: as a general approach for depressive relapse prevention MBCT was found to be not more effective than the same programme but without the mindfulness meditation component (Williams et al. 2014). For understanding if and how mindfulness meditation practices (rather than multi-component interventions) can foster cognitive enhancement, such limitations in evidence will need to be taken into account. Therefore, it is important to distinguish between outcomes from complex, multi-component programmes such as MBSR or MBCT from studies that isolated "pure" effects of specific meditation practices.

The aim of this article is to offer a conceptual and structural framework for the hypothesised neuroprotective effects of MM in cognitive ageing and to propose mechanisms by which MM may contribute to healthier cognitive ageing and to the preservation of cognitive functions with ageing. In the next section, the article includes a summary of recent interpretations regarding the role of cognitive functions in MM. This is followed by a section proposing that MM integrates two different types of brain training, namely brain network and brain state training. Furthermore, this section considers potential near and far transfer effects when discussing the extent to which MM leads to generalised cognitive improvements beyond the specific functions that are engaged during meditation. In the subsequent section "Ageing, Lifestyle and Cognitive Reserve", these considerations are applied to the cognitive reserve hypothesis, asking in what ways MMbased brain state and brain network training may contribute to preserving cognitive functions in older age. Following on from this, the section "Meditation, Ageing and Cognitive
Functions" reviews the currently rather limited empirical evidence and relates it to the theoretical propositions. This is rounded off by research suggestions on how robust empirical evidence regarding mindfulness and cognitive reserve can be accumulated.

\section{Mindfulness Meditation and Cognitive Skill Training}

Within current psychological and neuroscientific discourse, mindfulness is usually defined as a mental state characterised by "paying attention on purpose, in the present moment, and nonjudgmentally to the unfolding of experience moment by moment" (Kabat-Zinn 2003). Importantly, the ability to achieve and maintain mindful mental states can be developed through targeted mindfulness meditation (MM) practices (Malinowski 2008).

Bishop et al. (2004) introduced a concise model of MM, proposing self-regulation of attention and orientation to experience as core processes. Shapiro et al. (2006) expanded this model by including intention as a third component. According to their intention-attention-attitude model, three components dynamically interact during MM: (1) personal vision, purpose or intention; (2) paying close attention to momentary internal and external experience; and (3) attending to experience with an accepting, open and kind attitude. By emphasising the role of body sensations as objects of attention during MM, Hölzel et al. (2011) present a slightly different position, which reframes Shapiro et al.'s attitudinal component in terms of different emotion regulation skills. The Liverpool Mindfulness Model (Malinowski 2012, 2013) integrates these different perspectives by organising them into five tiers: (1) motivational factors including intention, (2) mind training, (3) mental core processes, (4) the mental/attitudinal stance and (5) the outcomes in terms of physical and mental well-being and associated behavioural changes. More traditional accounts of MM add an important perspective to these contemporary psychological models. Buddhist meditation instructions emphasise that with increasing experience the required effort decreases. Initially effort is required to balance between all forms of mental agitation and laxity which are eventually completely overcome (Wallace 1999). The sixteenth century master Karmapa Wangchug Dorje (2009) explained this process in more detail by referring to nine stages of mental stability that have been described in a famous meditation manual composed by the eighth century Indian scholar Kamalaśîla (1997). Contemporary accounts are provided, for example, by Jampa Thaye (2016) or Wallace (2005). In his meditation manual "Clarifying the Natural State" the sixteenth century scholar Dakpo Tashi Namgyal summarises the development of attentional stability into three main stages, figuratively comparing levels of mental agitation to a "mountain stream rolling through a gorge", a "placid flow 
of a river" and finally "the mother ocean meeting its river child" (Dakpo Tashi Namgyal 2001, p. 26).

Although each model emphasises different aspects, they all agree that attention is a core component of MM. To differentiate the involved attentional functions and their underlying brain networks, Malinowski (2013) utilises the attention model introduced by Posner and co-workers (Posner 2012; Posner and Rothbart 2007). During meditation, the alerting network establishes and sustains alertness, the salience and the executive control networks monitor and control mental states and recognise mind wandering or distraction and the orienting network disengages from distraction and redirects the attention focus back to the object of meditation. The underlying assumption is that repeated activation of these networks during meditation practice will strengthen their functioning and in consequence improve attentional abilities. These attentional functions are furthermore intertwined with working memory, the capacity to retain and manipulate goal-relevant information without distraction (Jha et al. 2010; Mrazek et al. 2013). During MM working memory is required, for example, to keep the meditation object and/or instruction in mind throughout the practice.

Recent reviews summarise the evidence that MM positively impacts on a range of cognitive functions, including sustained attention, executive or attentional control and working memory (Chiesa et al. 2011; Lippelt et al. 2014). These improvements in cognitive performance appear quite remarkable when compared to typical cognitive training effects. Typically the enhancement of cognitive skills only transfers to tasks, stimuli or contents that are quite similar to the ones trained (Green and Bavelier 2008; Klingberg 2010; MelbyLervåg and Hulme 2013). In contrast to such near transfer effects, cognitive enhancement resulting from MM practice appears to demonstrate far transfer (for a taxonomy of near and far transfer, see Barnett and Ceci 2002). That is, by engaging in MM practices that are concerned with internal experiences such as breath-related physical sensations, improvements in rather different cognitive tasks are achieved.

With focus on MM processes Tang and Posner proposed an alternative classification of training effects by distinguishing between brain network training and brain state training (Tang and Posner 2009, 2014). Whereas brain network training would improve cognitive abilities by repeatedly engaging the brain networks that are involved in specific cognitive functions, state training would involve more general brain states and activate large-scale brain networks that underpin a whole range of cognitive and emotional processes. These two types of training would also differ in terms of effort. Brain network training is thought to be consistently effortful. As meditation involves brain state training, it would only initially require a larger degree of effort to achieve and maintain meditation states. This effort would subsequently reduce as levels of expertise increase. Echoing traditional meditation accounts (e.g.
Dakpo Tashi Namgyal 2001) and establishing and maintaining a meditation state would be virtually effortless for advanced meditators (Tang et al. 2012).

Although the classification of training according to near vs far transfer or according to network vs state training differ in focus, it is useful to take both into account when considering the trajectory of cognitive enhancement effects resulting from MM training.

At the beginning, when a person starts to engage in MM training, establishing and maintaining the attentional focus will require effort and will engage the different attention networks (Malinowski 2013). An elegant functional magnetic resonance imaging (fMRI) study demonstrates how these networks are involved during meditation and how brain activity varies with expertise: When meditators re-established a meditation state by shifting back from mind wandering to meditation focus, different brain networks related to attentional functions were consecutively engaged. Levels of brain activity in these attention networks were lower the more lifetime meditation experience a participant had clocked up, supporting the proposition that less effort is required with growing expertise (Hasenkamp et al. 2012). In an electroencephalography (EEG) study, meditators were subjected to distracting sounds while engaged in MM practice. Several lines of analysis indicated increased perceptual clarity and decreased automated reactivity during practice compared to a control period of free thought (Cahn et al. 2010, 2012; Cahn and Polich 2009). Thus, both studies demonstrate superior functioning of attentional networks during meditation. As in both studies the investigated neural processes are either identical (Hasenkamp et al.) or very similar (Cahn et al.), they constitute good examples of near transfer effects, most likely resulting from brain network training. The observation that with growing expertise less effort (indexed by reduced brain activity) is required to reinstate a meditative state may indicate some brain state training effects. However, because up to now only very few studies investigated the dynamics of meditation states, evidence for the involvement of specific brain networks, or of network training and state training effects, is very limited.

Comparably, more extensive research shows that engaging in meditation practice results in improved cognitive performance, which is associated with changes in brain activity indicative of improved neural efficiency (Chiesa et al. 2011; Lutz et al. 2008; Malinowski 2013; Slagter et al. 2011). The cognitive enhancement observed in these studies by and large reflects far transfer because effects are found in tasks that are very different to what is practiced during MM.

However, to what extent these improvements reflect brain network or brain state effects is less clear. On the one hand, the attentional control processes involved in establishing, sustaining and re-establishing the meditative focus during MM practice engages various attention networks. In this sense, MM 
practice can certainly be classed as brain network training. On the other hand, MM practice also appears to be associated with more generic effects linked to entering and maintaining meditation-related global states of awareness, indicating brain state training. According to Tang and Posner, these meditation states are associated with changes to functions of the autonomic nervous system (ANS), which they consider central to brain state training (Tang and Posner 2009, 2014). However, when considering associated evidence it is important to acknowledge that effects on ANS function strongly depend on the type of meditation. For example, a comparison of expert meditators from different Buddhist meditation traditions revealed that Theravada and Vajrayana types of meditation had opposite effects on ANS functions (Amihai and Kozhevnikov 2014). Theravada meditation stimulated the parasympathetic nervous system (PNS) which supports the "rest and digest" functions of the body. In contrast, Vajrayana meditation activated the sympathetic nervous system (SNS), which increases metabolism and alertness and induces the "fight or flight" state. In line with this, the study demonstrated improvements in cognitive tasks immediately after Vajrayana but not after Theravada meditations, potentially indicating that the Vajrayana practice increased phasic alertness. Similarly, when meditation novices engaged over a 3month period in either breath awareness, thought awareness or loving-kindness meditation differential effects on ANS functions ensued (Lumma et al. 2015).

The picture is further complicated by the fact that many MM programmes also include physical exercises that may influence ANS function. The hormone cortisol is secreted in response to activation of the hypothalamic-pituitaryadrenocortical (HPA) axis and the SNS (Ulrich-Lai and Herman 2009). Increased cortisol levels are often used as a marker of stress and supressed immune function (McGregor et al. 2016). Furthermore, studies have shown that moderate and high-intensity exercise increases circulating cortisol levels, whereas low-intensity exercise reduces them (Hill et al. 2008). Therefore, physical exercise elements in complex MM programmes may have effects on cortisol levels that are independent from the effect of the meditation components and may differ depending on the type of exercise.

Tang and Posner based their arguments regarding brain state training primarily on research into the effects of integrative mind-body training (IBMT). In addition to meditation exercises, IBMT also involves body relaxation, manipulation of the breath and mental imagery (Tang et al. 2007). By contrasting IBMT with body relaxation exercises as active control condition, the explanation of mere relaxation effects is ruled out. Nevertheless, the extent to which the reported influence of IBMT on ANS function (Tang et al. 2009) stems from meditation practice alone, or from combining it with imagery, physical and respiratory exercises awaits further investigation. In any case, when considering $\mathrm{MM}$ as brain state training, it needs to be acknowledged that different MM regimes may have quite different effects on the ANS and the HPA axis.

In addition to the specific effects of MM on task-specific brain networks (network training) and the more global effects of state training on ANS functions, global influences on more generic cognitive functions and associated large-scale networks require consideration. The recent proposition of a generic multiple demand system of frontal and parietal brain areas that coordinates the involvement of task-specific networks (Duncan 2010) may play an important role in this respect (Raffone and Srinivasan 2017). More precisely, while establishing and maintaining mindful meditation states is likely to involve task-specific attention functions (Malinowski 2013), their activation and interplay during MM may be orchestrated by the multiple demand system and its phasic recruitment of task-specific networks (Duncan 2013; Fedorenko et al. 2013). Because a core feature of MM training is to be aware of domain-specific content such as thoughts, feelings and physical sensations without elaboration, the enhancement of neurocognitive meta-flexibility (Raffone and Srinivasan 2017) through activation of a domain-general, multiple purpose system seems plausible. As the multiple demand network is thought to flexibly engage a wide range of cognitive functions, it may play a crucial role in MM by underpinning several of the observed far transfer effects. The fact that there is significant overlap between the salience network and some areas of the multiple domain network may be key. The salience network is involved in detecting motivationally relevant or salient events across modalities (cognitive, homeostatic or emotional) in accordance with current goals (Dosenbach et al. 2007; Seeley et al. 2007) and fulfils this function also in terms of attentional engagement during MM (Hasenkamp et al. 2012). Because it interacts closely with ANS function (Critchley et al. 2013; Menon and Uddin 2010), it may be pivotal for brain state training during MM. The interaction of the salience network with the multiple domain system and the ANS may thus account for far transfer effects observed as a result of MM.

Given that MM practice involves the refinement of attention, the flexible engagement and disengagement of metacognitive functions and the balancing of arousal and laxity, it is likely that brain network and brain state training are involved. Improving the attentional focus and stability will lead to enhancement of attentional brain networks (brain network training). The dynamic engagement of cognitive operations will lead to enhanced functioning of the multiple demand network and the balancing of alertness levels will influence brain networks associated with alertness and arousal (brain state training). Because the effort required for engaging in MM decreases as experience increases, the balance may shift from primarily brain network training that strengthens specific cognitive functions towards refinement of meditation states by means of brain state training. 
Therefore, it would be important to specify the expected brain network training effects, as well as brain state training effects when investigating the effects of MM training. To start disentangling the influences of network and state training empirical studies should ideally include measures for quantifying both. Thus, future research may investigate whether different meditation practices rely more heavily on one or the other type of training and whether this balance shifts with increasing expertise. As the invested effort influences behavioural performance (Jensen et al. 2012) and neural activity (Brefczynski-Lewis et al. 2007) and autonomic functioning is associated with cognitive performance, particularly in older adults (Frewen et al. 2013), it will be important to control such variables when considering cognitive enhancement effects of meditation

\section{Ageing, Lifestyle and Cognitive Reserve}

The effects of various cognitive training programmes that have been developed for healthy older adults and people with mild cognitive impairments (MCI), a common precursor of Alzheimer's disease, seem to be limited to near transfer effects with very little, if any, generalisation to overall cognitive functioning and daily life situations (Reijnders et al. 2013). Because MM practice has been demonstrated to yield a range of far transfer effects, it may offer an attractive alternative. Based on reported associations between ANS dysfunctions and cognitive performance in older adults (Frewen et al. 2013), we may furthermore speculate that the brain state training component positively impacts cognitive performance by improving autonomic functioning.

A significant body of epidemiological evidence suggests that the degree of age-related cognitive decline is influenced by a range of non-biological lifestyle factors, such as level of education (Meng and D'Arcy 2012), occupational complexity (Dekhtyar et al. 2015) and leisure activities (Scarmeas and Stern 2003). A considerable number of older people demonstrate far better cognitive performance than their level of brain pathology predicts, which has been explained by the cognitive reserve hypothesis (Stern 2002, 2003, 2009). This hypothesis specifies that cognitive functions are preserved due to two different processes (Steffener and Stern 2012). Neural reserve is the process of sustaining cognitive performance due to higher network efficiency, capacity or flexibility, akin to the mechanism by which healthy individuals cope with increased difficulty or task demands. Neural compensation refers to the process of recruiting alternative brain networks and structures that are not normally engaged in a certain function. For example, older adults who recruit bilateral (rather than unilateral) prefrontal areas perform better in cognitive tasks than older adults who do not recruit additional brain regions (Cabeza et al. 2002; Langenecker and Nielson 2003; Nielson et al.
2002; Piefke et al. 2012). Such compensatory processes may be enlisted when primary neural networks are no longer able to adequately support successful task performance (ReuterLorenz 2002; Steffener et al. 2009), although performance may not be fully maintained.

Based on the evidence that lifestyle factors can contribute to cognitive reserve and on the observed far transfer effects of $\mathrm{MM}$, we propose that MM may indeed enhance cognitive reserve capacity (see also Xiong and Doraiswamy 2009), potentially even in older individuals suffering from mild cognitive impairment (Larouche et al. 2015; Wells et al. 2013). The structure of this proposition is depicted in Fig. 1. Brain network training may activate the brain areas involved in attention (Malinowski 2013), working memory (Mrazek et al. 2013) and other specific cognitive processes, thus directly enhancing neural reserve. The cognitive aspect of brain state training would lead to an enhancement of the large-scale multiple demand system (Raffone and Srinivasan 2017), as second direct pathway of enhancing neural reserve. The balancing and stress-reducing effects of MM may positively influence ageing by improving functioning of ANS (Tang et al. 2009), HPA axis and immune functions (Creswell et al. 2012; Epel et al. 2009; Epel et al. 2013; Larouche et al. 2015). As all three factors have been implicated in decline of brain structure (Frewen et al. 2013; Larouche et al. 2015; Lupien et al. 2009; McEwen and Magarinos 2001; Simen et al. 2011), the brain state training of MM may also enhance neural reserve via this indirect pathway.

\section{Meditation, Ageing and Cognitive Functions}

Although direct, robust evidence that meditation leads to better cognitive health in older adults is virtually non-existent, a

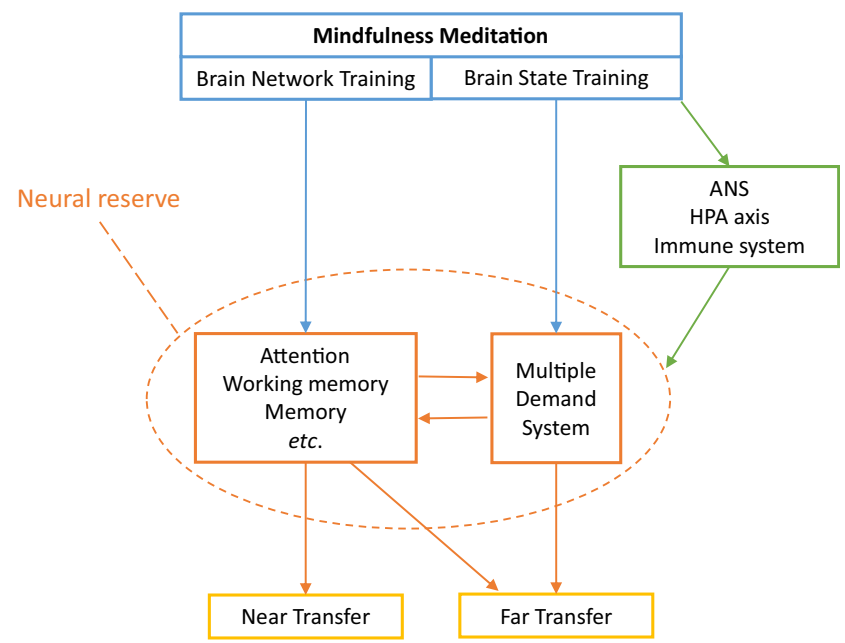

Fig. 1 The proposed key mechanisms relating mindfulness meditation to different types of training, functional improvements, neural reserve and resulting transfer effects 
number of studies provide encouraging, yet indirect, results. Older meditators performed better than age-matched nonmeditators on the attentional blink task and their performance was comparable to that of a young control group (van Leeuwen et al. 2009). The attentional blink task assesses the efficiency of allocating attentional resources over time and performance is typically reduced with age (GeorgiouKaristianis et al. 2007; Maciokas and Crognale 2003). Similarly, performance on a sustained attention task was negatively related to age in a matched control group, but not in a group of experienced meditators who performed as well as their younger counterparts (Pagnoni and Cekic 2007). In line with these performance data, only the control group displayed the negative correlation between grey matter volume and age that is indicative of cognitive ageing. This effect was most pronounced in the left putamen, which has been implicated in cognitive flexibility and attentional processing (Nieoullon 2002). Comparing cortical thickness between a group of longterm meditators and age-matched controls, Lazar et al. (2005) found that age-related decreases in right frontal regions were only present in the control group. In contrast, cortical thickness of older meditators was comparable to that of younger meditators. In a study on differences in fractional anisotropy (FA), an index of white matter integrity (Madden et al. 2012; Madden et al. 2009), Luders et al. (2011) reported that the agerelated FA decrease was less pronounced in meditators than in controls. These cross-sectional studies provide a first indication that MM might protect against cognitive decline. Preserved cognitive performance may indicate far transfer effects, whereas preserved neural structure may be an indicator of neural reserve. Because these cross-sectional studies do not demonstrate causal relationships, longitudinal approaches are required to corroborate the evidence.

In the last few years, a small number of studies investigated more directly whether MM can improve cognitive performance in older adults. Two studies have examined the effects of 7- to 8-week-long mindfulness-based programmes on cognitive functions in older adults with normal cognitive functioning. An adapted 7-week version of the MBSR/MBCT intervention for older dementia caregivers (mean age 65 years) did not show any mindfulness-specific improvements in cognitive functions in comparison to an active control group (Oken et al. 2010). The second study demonstrated that at the end of an 8-week MBSR programme, a group of healthy older adults (mean age 73 years) improved on a measure of executive functions, as compared to a waitlist control group (Mallya and Fiocco 2015). However, this effect was not maintained 3 and 24 weeks post intervention. A pilot study with a small number of adults with MCI found that MBSR increased functional connectivity between the posterior cingulate cortex (PCC), medial prefrontal cortex (MPFC) and left hippocampus and trends of less hippocampal atrophy (Wells et al. 2013). Because the hippocampus is strongly affected by
ANS and HPA axis functioning, this effect may reflect brain state training. The increased functional connectivity between areas that are part of the default mode network (DMN) may indicate network training effects, although there is conflicting evidence whether MM would lead to an increase or decrease of DMN connectivity (Jang et al. 2011; Taylor et al. 2013). Indeed, some evidence suggests that connectivity is increasing between some areas while decreasing between others (Brewer et al. 2011).

However, all these studies investigated the effects of multicomponent interventions. They are thus relatively uninformative regarding the specific effects of $\mathrm{MM}$ on cognitive ageing. To avoid such ambiguity, we thus investigate the effects of a single, well-defined mindful breath awareness practice, rather than a conglomerate of programme components (Malinowski et al. 2017; Moore et al. 2012). Over a period of 8 weeks, healthy, older participants (mean age 65 years) engaged in mindful breath awareness training (M-BAT), 10 min daily, five times per week. They were compared to an active control group who engaged with the same dosage of commercially available arithmetic exercises ("brain gym"). We assessed changes in cognitive processes with a range of computerised cognitive tests combined with concurrent EEG recordings. The results showed that compared to the active control group, MM practice improved general task performance, indexed by reduced response times and increased $\mathrm{N} 2$ event-related potential (ERP) amplitudes in an emotional counting Stroop task, potentially reflecting improvements in the maintenance of goal-directed visuospatial attention (Malinowski et al. 2017). Furthermore, in a response inhibition task (continuous performance task), which assesses the ability to inhibit a prepotent motor responses, MM selectively reduced response times and enhanced P3 ERP amplitudes in inhibition trials (nogo trials), indexing improved executive attention skills (Malinowski et al. Mindful ageing: mindful breath awareness practice improves inhibitory control in older adults, in preparation). Thus, when older adults start engaging in M-BAT, initially improvements manifest in performance and neural activity that reflect far transfer from breath awareness to cognitive tasks and arguably brain network training. Because both groups expected cognitive improvement and both were as actively engaged in their practices, we can rule out confounding influences of effort. However, as we were not able to include measures of ANS or HPA axis functioning, we cannot say whether in addition to the observed network training effects also more general effects resulting from state training appeared.

\section{Conclusions}

Before drawing conclusions, we shall acknowledge that research into mindfulness is still in its infancy and that at present the vast majority of claims presented in the literature need to be considered preliminary. For the complexity of the matter, 
the available amount of evidence is too limited to allow robust conclusions, even for the most extensively researched applications of mindfulness. This applies even more to the questions whether MM can improve cognitive functions associated with ageing, if it can limit or prevent age-related cognitive decline or if it can even restore cognitive functions.

Limited cross-sectional evidence of retained cognitive performance, neural activity and neural structure suggests that MM may protect against age-related decline. While crosssectional studies per se are limited in attributing causality, this issue is particularly pertinent when considering age-related effects. It is highly likely that these studies suffer from a selection bias. For example, we have to assume that only meditators who did not develop dementia or other age-related deficits will participate in the research, whereas all those who were not able to maintain their meditation practice will not.

Evidence from intervention studies is even sparser. A few studies investigated the effects of different MM programmes in older adults, either with normal/healthy cognitive functions or with MCI. Some evidence of improvements of cognitive functions and underlying neural processes was evident, but given the paucity of studies and the large variety of approaches, it is impossible to draw reliable conclusions.

It is worth noting that these studies were largely limited to short-term effects of up to 8 weeks, measured directly after the end of the intervention. In the only study that included followup measurements, the enhanced executive functions were not maintained at 3 and 24 weeks post intervention (Mallya and Fiocco 2015). So far, these studies merely show that some MM programmes can enhance cognitive performance in older adults. Whether they improve cognitive reserve capacity is currently unknown. Because all known factors that contribute to cognitive reserve capacity exert their influence over long time periods (e.g. education or occupation), it is reasonable to expect that also for MM long-term engagement will be required to yield lasting effects. This means that standard 8week high-intensity programmes such as MBSR or MBCT may not be well suited for a sustained approach. Different, less prescriptive approaches delivered by highly experienced mindfulness meditation teachers with the capability to operate outside of these standard programmes may be required (Malinowski 2017). It also means that research programmes with a long-term perspective are required to answer questions about cognitive reserve capacity.

Furthermore, the interpretation of cross-sectional and intervention studies into MM is limited by the fact that a broad range of interventions and meditation traditions have been examined, sometimes without sufficient differentiation between the type of intervention, the specific meditation tradition or the specific meditation exercise. Due to a lack of replication studies, we currently do not know whether the sometimes contradictory findings are due to differences in intervention or meditation style or merely due to statistical type 1 errors.
Despite all these limitations, the existing evidence is promising (Gard et al. 2014; Luders 2014). The positive results that several studies yield allow us to be confident that long-term research programmes are feasible.

Research into the cognitive effects of mindfulness meditation indicates that far transfer effects occur, making MM an attractive candidate for the enhancement of cognitive reserve capacity. We can furthermore assume that these far transfer effects are a combined result of brain network training and brain state training. Whereas the network training enhances specific cognitive functions involved in MM, state training enhances the large-scale multiple demand system and exerts indirect influence by improving the response functions of ANS, HPA axis and immune system. These improvements in turn have a positive influence on brain structure, such as the hippocampus. Thus, we hypothesised that network training and state training will contribute to neural reserve via direct and indirect routes, respectively.

The growing evidence that MM improves ANS, HPA axis and immune function (Black and Slavich 2016; O'Leary et al. 2016; Tang et al. 2009) is in itself exciting, indicating that it holds promise beyond cognitive improvements. Even if cognitive enhancement turns out to be less pronounced than expected or hoped for, the reduction in psychological stress that is assumed to lead to improvements in physiological functioning is worthwhile. The absence of side effects of MM compared to pharmacological interventions for dementia that are currently under investigation (Herholz et al. 2013) further underlines the promise of MM.

\section{Recommendations for Future Research}

To move research into the neuroprotective potential of mindfulness meditation forward several recommendations should be considered:

Randomised Active Controlled Trials To establish causal mechanisms, randomised controlled trials with active control groups are indispensable. The control activities should be matched as closely as possible to the experimental condition. Ideally, they will only differ in terms of the meditation component, whereas all other aspects are identical. The activity replacing the meditation component should be as similar as possible to meditation, without being meditation. A high standard would have been achieved if the participants in the active control condition were not aware that they are control participants and expect outcomes comparable to the experimental group.

Long-Term Perspective It will be particularly important to conduct studies of considerable length, so that effects on cognitive reserve capacity can be evaluated. This long-term 
perspective should also be applied when designing programmes or interventions. Rather than relying on compact 8 -week interventions, approaches that are feasible over long time periods and that support participants in embedding their practice within their daily routine should be considered. Related to this, the question of long-term take up and maintenance of MM practice would be relevant. Ideally, participants would be followed over many years to gain insight into the long-term trajectories of practice engagement and resulting effects.

Tailored Programmes The primarily preventative nature of this approach asks for a curriculum that differs from the typical ameliorative programmes which rely on a medical treatment model. Offers would need to be tailored to the specific needs and life circumstances of older individuals and support them in becoming self-reliant in the maintenance of their MM practice. In addition, workplace offers or community-based self-help programmes should be considered.

Clear Definitions and Programme Descriptions Some uncertainty in the literature results from the fact that a range of different programmes and approaches are lumped together as mindfulness meditation. A clear description of the implemented programme that could follow emerging classification systems would facility the integration of results across studies.

Practitioner Information To account for potential differences in expertise and approach, detailed information about qualification of mindfulness practitioners facilitating a programme and about their levels of expertise would be meaningful.

Isolation of the Active Meditation Component To develop our understanding of the contribution of individual mindfulness meditation practices, it will be crucial to examine them in isolation, rather than embedding them within a conglomerate of different exercises. Knowing the effects of individual components is a prerequisite for tailoring programmes to individual requirements and conditions.

Specifying Brain Network and State Training Effects In this article, we developed the proposition of these two forms of training further and suggested that they may work in conjunction, potentially with different emphasis and a shift of balance over time. To investigate the involvement of these types of training, it will be important to specify the intended effects precisely, for example, in terms of arousal levels, effort, involved cognitive functions and networks, etc. Change measures should ideally include some that capture network training effects (behavioural, brain activity and structure) while others should capture aspects of state training effects on
ANS functioning (e.g. heart rate variability), HPA axis (e.g. cortisol) and immune function (e.g. interleukin 6).

Prospective Cohort Studies Studies that follow a large, representative sample of participants for long time periods would be ideal. Given that only very few individuals engage in regular meditation practice makes this approach currently not feasible. Nevertheless, we should keep in mind that without such studies evidence regarding the actual, real-life neuroprotective potential of MM will remain limited. Long-term randomised active controlled trials will go some way towards this end, but cannot fully replace such data from cohort studies.

As this extensive, albeit incomplete list demonstrates, the thorough investigation of the most important questions demands patience. Researchers and practitioners should approach this mindfully, cultivating such patience - one central pillar of MM practice (Kabat-Zinn 1990). We should resist the temptation to succumb to the current mindfulness boom and rather develop our understanding step-by-step until we can say with some certainty which mindfulness approach fits for which person under which conditions, how much practice will be required for how long to enhance cognitive reserve capacity and yield neuroprotective effects.

Open Access This article is distributed under the terms of the Creative Commons Attribution 4.0 International License (http:// creativecommons.org/licenses/by/4.0/), which permits unrestricted use, distribution, and reproduction in any medium, provided you give appropriate credit to the original author(s) and the source, provide a link to the Creative Commons license, and indicate if changes were made.

\section{References}

Albert, S. M., Glied, S., Andrews, H., Stern, Y., \& Mayeux, R. (2002). Primary care expenditures before the onset of Alzheimer's disease. Neurology, 59(4), 573-578.

Amihai, I., \& Kozhevnikov, M. (2014). Arousal vs. relaxation: a comparison of the neurophysiological and cognitive correlates of Vajrayana and Theravada meditative practices. Public Library of Science One, 9(7), e102990. doi:10.1371/journal.pone.0102990.

Barnett, S. M., \& Ceci, S. J. (2002). When and where do we apply what we learn?: a taxonomy for far transfer. Psychological Bulletin, $128(4), 612-637$.

Bishop, S. R., Lau, M. A., Shapiro, S. L., Carlson, L. E., Anderson, N. D., Carmody, J., et al. (2004). Mindfulness: a proposed operational definition. Clinical Psychology: Science and Practice, 11(3), 230-242. doi:10.1093/clipsy.bph077.

Black, D. S., \& Slavich, G. M. (2016). Mindfulness meditation and the immune system: a systematic review of randomized controlled trials. Annals of the New York Academy of Sciences, 1373(1), 13-24. doi: 10.1111/nyas.12998.

Brefczynski-Lewis, J. A., Lutz, A., Schaefer, H. S., Levinson, D. B., \& Davidson, R. J. (2007). Neural correlates of attentional expertise in long-term meditation practitioners. Proceedings of the National 
Academy of Sciences of the USA, 104(27), 11483-11488. doi:10. 1073/pnas.0606552104.

Brewer, J. A., Worhunsky, P. D., Gray, J. R., Tang, Y. Y., Weber, J., \& Kober, H. (2011). Meditation experience is associated with differences in default mode network activity and connectivity. Proceedings of the National Academy of Sciences, 108(50), 20254-20259.

Cabeza, R., Anderson, N. D., Locantore, J. K., \& McIntosh, A. R. (2002). Aging gracefully: compensatory brain activity in high-performing older adults. NeuroImage, 17(3), 1394-1402. doi:10.1006/nimg. 2002.1280 .

Cahn, B. R., Delorme, A., \& Polich, J. (2010). Occipital gamma activation during Vipassana meditation. Cognitive Processing, 11(1), 3956. doi:10.1007/s10339-009-0352-1.

Cahn, B. R., Delorme, A., \& Polich, J. (2012). Event-related delta, theta, alpha and gamma correlates to auditory oddball processing during Vipassana meditation. Social Cognitive and Affective Neuroscience. doi:10.1093/scan/nss060.

Cahn, B. R., \& Polich, J. (2009). Meditation (Vipassana) and the P3a eventrelated brain potential. International Journal of Psychophysiology, 72(1), 51-60. doi:10.1016/j.ijpsycho.2008.03.013.

Chiesa, A., Calati, R., \& Serretti, A. (2011). Does mindfulness training improve cognitive abilities? A systematic review of neuropsychological findings. Clinical Psychology Review, 31(3), 449-464. doi: 10.1016/j.cpr.2010.11.003.

Chiesa, A., \& Serretti, A. (2014). Are mindfulness-based interventions effective for substance use disorders? A systematic review of the evidence. Substance Use \& Misuse, 49(5), 492-512. doi:10.3109/ 10826084.2013.770027.

Colcombe, S. J., Erickson, K. I., Scalf, P. E., Kim, J. S., Prakash, R., McAuley, E., et al. (2006). Aerobic exercise training increases brain volume in aging humans. The Journals of Gerontology. Series A, Biological Sciences and Medical Sciences, 61(11), 1166-1170.

Creswell, J. D., Irwin, M. R., Burklund, L. J., Lieberman, M. D., Arevalo, J. M., Ma, J., et al. (2012). Mindfulness-based stress reduction training reduces loneliness and pro-inflammatory gene expression in older adults: a small randomized controlled trial. Brain, Behavior, and Immunity, 26(7), 1095-1101. doi:10.1016/j.bbi.2012.07.006.

Critchley, H. D., Eccles, J., \& Garfinkel, S. N. (2013). Interaction between cognition, emotion, and the autonomic nervous system. In R. M. Buijs \& D. F. Swaab (Eds.), Handbook of clinical neurology; Autonomic nervous system (Vol. 117, pp. 59-77): Elsevier.

Dakpo Tashi Namgyal. (2001). Clarifying the natural state: A principal guidance manual for Mahamudra: Transl. into English by Erik Pema Kunsang. Hong Kong: Rangjung Yeshe.

DeCarli, C. (2003). Mild cognitive impairment: prevalence, prognosis, aetiology, and treatment. Lancet Neurology, 2(1), 15-21.

Dekhtyar, S., Wang, H. X., Scott, K., Goodman, A., Koupil, I., \& Herlitz, A. (2015). A life-course study of cognitive reserve in dementifrom childhood to old age. American Journal of Geriatric Psychiatry, 23(9), 885-896. doi:10.1016/j.jagp.2015.02.002.

Dosenbach, N. U., Fair, D. A., Miezin, F. M., Cohen, A. L., Wenger, K. K., Dosenbach, R. A., et al. (2007). Distinct brain networks for adaptive and stable task control in humans. Proceedings of the National Academy of Sciences of the USA, 104(26), 11073-11078. doi:10.1073/pnas.0704320104.

Draganski, B., Gaser, C., Busch, V., Schuierer, G., Bogdahn, U., \& May, A. (2004). Neuroplasticity: changes in grey matter induced by training. Nature, 427(6972), 311-312. doi:10.1038/427311a.

Driemeyer, J., Boyke, J., Gaser, C., Buchel, C., \& May, A. (2008). Changes in gray matter induced by learning-revisited. Public Library of Science One, 3(7), e2669. doi:10.1371/journal.pone. 0002669 .

Duncan, J. (2010). The multiple-demand (MD) system of the primate brain: mental programs for intelligent behaviour. Trends in Cognitive Sciences, 14(4), 172-179. doi:10.1016/j.tics.2010.01.004.
Duncan, J. (2013). The structure of cognition: attentional episodes in mind and brain. Neuron, 80(1), 35-50. doi:10.1016/j.neuron.2013.09.015.

Epel, E., Daubenmier, J., Moskowitz, J. T., Folkman, S., \& Blackburn, E. (2009). Can meditation slow rate of cellular aging? Cognitive stress, mindfulness, and telomeres. Annals of the New York Academy of Sciences, 1172, 34-53. doi:10.1111/j.1749-6632.2009.04414.x.

Epel, E., Puterman, E., Lin, J., Blackburn, E., Lazaro, A., \& Mendes, W. B. (2013). Wandering minds and aging cells. Clinical Psychological Science, 1(1), 75-83. doi:10.1177/2167702612460234.

Ernst, R. L., \& Hay, J. W. (1997). Economic research on Alzheimer disease: a review of the literature. Alzheimer Disease and Associated Disorders, 11(Suppl 6), 135-145.

Fedorenko, E., Duncan, J., \& Kanwisher, N. (2013). Broad domain generality in focal regions of frontal and parietal cortex. Proceedings of the National Academy of Sciences, 110(41), 16616-16621.

Frewen, J., Finucane, C., Savva, G. M., Boyle, G., Coen, R. F., \& Kenny, R. A. (2013). Cognitive function is associated with impaired heart rate variability in ageing adults: the Irish longitudinal study on ageing wave one results. Clinical Autonomic Research, 23(6), 313-323. doi:10.1007/s10286-013-0214-x.

Gage, F. H. (2002). Neurogenesis in the adult brain. The Journal of Neuroscience, 22(3), 612-613.

Gard, T., Hölzel, B. K., \& Lazar, S. W. (2014). The potential effects of meditation on age-related cognitive decline: a systematic review. Annals of the New York Academy of Sciences, 1307, 89-103. doi: 10.1111/nyas.12348.

Georgiou-Karistianis, N., Tang, J., Vardy, Y., Sheppard, D., Evans, N., Wilson, M., et al. (2007). Progressive age-related changes in the attentional blink paradigm. Neuropsychology, Development, and Cognition. Section B, Aging, Neuropsychology and Cognition: A Journal on Normal and Dysfunctional Development, 14(3), 213226. doi:10.1080/13825580500320681.

Goyal, M., Singh, S., Sibinga, E. M., Gould, N. F., Rowland-Seymour, A., Sharma, R., et al. (2014). Meditation programs for psychological stress and well-being: a systematic review and meta-analysis. JAMA Internal Medicine, 174(3), 357-368. doi:10.1001/jamainternmed. 2013.13018.

Green, C. S., \& Bavelier, D. (2008). Exercising your brain: a review of human brain plasticity and training-induced learning. Psychology and Aging, 23(4), 692-701. doi:10.1037/a0014345.

Hasenkamp, W., Wilson-Mendenhall, C. D., Duncan, E., \& Barsalou, L. W. (2012). Mind wandering and attention during focused meditation: a fine-grained temporal analysis of fluctuating cognitive states. NeuroImage, 59(1), 750-760. doi:10.1016/j.neuroimage.2011.07.008.

Hedden, T., \& Gabrieli, J. D. (2004). Insights into the ageing mind: a view from cognitive neuroscience. Nature Reviews Neuroscience, 5(2), 87-96. doi:10.1038/nrn1323.

Herholz, S. C., Herholz, R. S., \& Herholz, K. (2013). Nonpharmacological interventions and neuroplasticity in early stage Alzheimer's disease. Expert Review of Neurotherapeutics, 13(11), 1235-1245. doi:10.1586/14737175.2013.845086.

Hill, E. E., Zack, E., Battaglini, C., Viru, M., Viru, A., \& Hackney, A. C. (2008). Exercise and circulating cortisol levels: the intensity threshold effect. Journal of Endocrinological Investigation, 31(7), 587-591.

Hofmann, S. G., Sawyer, A. T., Witt, A. A., \& Oh, D. (2010). The effect of mindfulness-based therapy on anxiety and depression: A metaanalytic review. Journal of Consulting and Clinical Psychology, 78(2), 169-183. doi:10.1037/a0018555.

Hölzel, B. K., Lazar, S. W., Gard, T., Schuman-Olivier, Z., Vago, D. R., \& Ott, U. (2011). How does mindfulness meditation work? Proposing mechanisms of action from a conceptual and neural perspective. Perspectives on Psychological Science, 6(6), 537-559. doi:10. 1177/1745691611419671.

Jampa Thaye, L. (2016). Diamond sky: A brief introduction to the Buddhist path (2nd ed.). Bristol: Ganesha Press. 
Jang, J. H., Jung, W. H., Kang, D. H., Byun, M. S., Kwon, S. J., Choi, C. H., \& Kwon, J. S. (2011). Increased default mode network connectivity associated with meditation. Neuroscience Letters, 487(3), 358-362. doi:10.1016/j.neulet.2010.10.056.

Jensen, C. G., Vangkilde, S., Frokjaer, V., \& Hasselbalch, S. G. (2012). Mindfulness training affects attention - or is it attentional effort? Journal of Experimental Psychology: General, 141(1), 106-123.

Jha, A. P., Stanley, E. A., Kiyonaga, A., Wong, L., \& Gelfand, L. (2010). Examining the protective effects of mindfulness training on working memory capacity and affective experience. Emotion, 10(1), 54-64. doi:10.1037/a0018438.

Kabat-Zinn, J. (1990). Full catastrophe living: using the wisdom of your body and mind to face stress, pain and illness. New York: Bantam Doubleday Dell Publishing.

Kabat-Zinn, J. (2003). Mindfulness-based interventions in context: Past, present, and future. Clinical Psychology: Science and Practice, 10(2), 144-156. doi:10.1093/clipsy.bpg016.

Kamalaśíla. (1997). Bhāvanākrama of Kamalaśila: Transl. into English by Parmananda Sharma. New Delhi: Aditya Prakashan.

Keng, S. L., Smoski, M. J., \& Robins, C. J. (2011). Effects of mindfulness on psychological health: a review of empirical studies. Clinical Psychology Review, 31(6), 1041-1056. doi:10.1016/j.cpr.2011.04.006.

Klingberg, T. (2010). Training and plasticity of working memory. Trends in Cognitive Sciences, 14(7), 317-324. doi:10.1016/j.tics.2010.05.002.

Langenecker, S. A., \& Nielson, K. A. (2003). Frontal recruitment during response inhibition in older adults replicated with fMRI. NeuroImage, 20(2), 1384-1392. doi:10.1016/s1053-8119(03) 00372-0.

Larouche, E., Hudon, C., \& Goulet, S. (2015). Potential benefits of mindfulness-based interventions in mild cognitive impairment and Alzheimer's disease: an interdisciplinary perspective. Behavioural Brain Research, 276, 199-212. doi:10.1016/j.bbr.2014.05.058.

Lazar, S. W., Kerr, C. E., Wasserman, R. H., Gray, J. R., Greve, D. N., Treadway, M. T., et al. (2005). Meditation experience is associated with increased cortical thickness. Neuroreport, 16(17), 1893-1897.

Lippelt, D. P., Hommel, B., \& Colzato, L. S. (2014). Focused attention, open monitoring and loving kindness meditation: effects on attention, conflict monitoring, and creativity - a review. Frontiers in Psychology, 5, 1083.

Luders, E. (2014). Exploring age-related brain degeneration in meditation practitioners. Annals of the New York Academy of Sciences, 1307, 82-88. doi:10.1111/nyas.12217.

Luders, E., Clark, K., Narr, K. L., \& Toga, A. W. (2011). Enhanced brain connectivity in long-term meditation practitioners. NeuroImage, 57(4), 1308-1316. doi:10.1016/j.neuroimage.2011.05.075.

Lumma, A. L., Kok, B. E., \& Singer, T. (2015). Is meditation always relaxing? Investigating heart rate, heart rate variability, experienced effort and likeability during training of three types of meditation. International Journal of Psychophysiology, 97(1), 38-45. doi:10. 1016/j.ijpsycho.2015.04.017.

Lupien, S. J., McEwen, B. S., Gunnar, M. R., \& Heim, C. (2009). Effects of stress throughout the lifespan on the brain, behaviour and cognition. Nature Reviews Neuroscience, 10(6), 434-445. doi:10.1038/ nrn2639.

Lutz, A., Slagter, H. A., Dunne, J. D., \& Davidson, R. J. (2008). Attention regulation and monitoring in meditation. Trends in Cognitive Sciences, 12(4), 163-169. doi:10.1016/j.tics.2008.01.005.

Lyketsos, C. G., Lopez, O., Jones, B., Fitzpatrick, A. L., Breitner, J., \& DeKosky, S. (2002). Prevalence of neuropsychiatric symptoms in dementia and mild cognitive impairment: results from the cardiovascular health study. The Journal of the American Medical Association, 288(12), 1475-1483.

Maciokas, J. B., \& Crognale, M. A. (2003). Cognitive and attentional changes with age: evidence from attentional blink deficits. Experimental Aging Research, 29(2), 137-153. doi:10.1080/ 03610730303715 .
Madden, D. J., Bennett, I. J., Burzynska, A., Potter, G. G., Chen, N. K., \& Song, A. W. (2012). Diffusion tensor imaging of cerebral white matter integrity in cognitive aging. Biochimica et Biophysica Acta, 1822(3), 386-400. doi:10.1016/j.bbadis.2011.08.003.

Madden, D. J., Bennett, I. J., \& Song, A. W. (2009). Cerebral white matter integrity and cognitive aging: contributions from diffusion tensor imaging. Neuropsychology Review, 19(4), 415-435. doi:10.1007/ s11065-009-9113-2.

Malinowski, P. (2008). Mindfulness as psychological dimension: concepts and applications. The Irish Journal of Psychology, 29(1-2), $155-166$.

Malinowski, P. (2012). Wirkmechanismen der Achtsamkeitspraxis. Wie fördert Meditation positive psychologische Veränderungen? In M. Zimmermann, C. Spitz, \& S. Schmidt (Eds.), Achtsamkeit: Ein buddhistisches Konzept erobert die Wissenschaft. Berne: Hans Huber.

Malinowski, P. (2013). Neural mechanisms of attentional control in mindfulness meditation. Frontiers in Neuroscience, 7, 8. doi:10.3389/ fnins.2013.00008.

Malinowski, P. (2017). Mindfulness. In S. Schneider \& M. Velmans (Eds.), The Blackwell companion to consciousness (2nd ed.). Oxford: Wiley.

Malinowski, P., Moore, A. W., Mead, B. R., \& Gruber, T. (2017). Mindful aging: the effects of regular brief mindfulness practice on electrophysiological markers of cognitive and affective processing in older adults. Mindfulness, 8(1), 78-94. doi:10.1007/s12671-015-0482-8.

Mallya, S., \& Fiocco, A. J. (2015). Effects of mindfulness training on cognition and well-being in healthy older adults. Mindfulness, 7(2), 453-465. doi:10.1007/s12671-015-0468-6.

McEwen, B. S., \& Magarinos, A. M. (2001). Stress and hippocampal plasticity: implications for the pathophysiology of affective disorders. Human Psychopharmacology: Clinical and Experimental, 16(S1), S7-S19.

McGregor, B. A., Murphy, K. M., Albano, D. L., \& Ceballos, R. M. (2016). Stress, cortisol, and B lymphocytes: a novel approach to understanding academic stress and immune function. Stress, 19(2), 185-191.

Melby-Lervåg, M., \& Hulme, C. (2013). Is working memory training effective? A meta-analytic review. Developmental Psychology, 49(2), 270-291. doi:10.1037/a0028228.

Meng, X., \& D'Arcy, C. (2012). Education and dementia in the context of the cognitive reserve hypothesis: a systematic review with metaanalyses and qualitative analyses. Public Library of Science One, 7(6), e38268. doi:10.1371/journal.pone.0038268.

Menon, V., \& Uddin, L. Q. (2010). Saliency, switching, attention and control: a network model of insula function. Brain Structure \& Function, 214(5-6), 655-667. doi:10.1007/s00429-010-0262-0.

Moore, A., Gruber, T., Derose, J., \& Malinowski, P. (2012). Regular, brief mindfulness meditation practice improves electrophysiological markers of attentional control. Frontiers in Human Neuroscience, 6, 18. doi:10.3389/fnhum.2012.00018.

Mrazek, M. D., Franklin, M. S., Phillips, D. T., Baird, B., \& Schooler, J. W. (2013). Mindfulness training improves working memory capacity and GRE performance while reducing mind wandering. Psychological Science, 24(5), 776-781. doi:10.1177/ 0956797612459659 .

Nielson, K. A., Langenecker, S. A., \& Garavan, H. (2002). Differences in the functional neuroanatomy of inhibitory control across the adult life span. Psychology and Aging, 17(1), 56-71.

Nieoullon, A. (2002). Dopamine and the regulation of cognition and attention. Progress in Neurobiology, 67(1), 53-83.

O’Leary, K., O’Neill, S., \& Dockray, S. (2016). A systematic review of the effects of mindfulness interventions on cortisol. Journal of Health Psychology, 21(9), 2108-2121. doi:10.1177/ 1359105315569095. 
Oken, B. S., Fonareva, I., Haas, M., Wahbeh, H., Lane, J. B., Zajdel, D., \& Amen, A. (2010). Pilot controlled trial of mindfulness meditation and education for dementia caregivers. Journal of Alternative and Complementary Medicine, 16(10), 1031-1038. doi:10.1089/acm. 2009.0733.

Pagnoni, G., \& Cekic, M. (2007). Age effects on gray matter volume and attentional performance in Zen meditation. Neurobiology of Aging, 28(10), 1623-1627. doi:10.1016/j.neurobiolaging.2007.06.008.

Piefke, M., Onur, O. A., \& Fink, G. R. (2012). Aging-related changes of neural mechanisms underlying visual-spatial working memory. Neurobiology of Aging, 33(7), 1284-1297. doi:10.1016/j. neurobiolaging.2010.10.014.

Piet, J., \& Hougaard, E. (2011). The effect of mindfulness-based cognitive therapy for prevention of relapse in recurrent major depressive disorder: a systematic review and meta-analysis. Clinical Psychology Review, 31(6), 1032-1040. doi:10.1016/j.cpr.2011.05.002.

Posner, M. I. (2012). Imaging attention networks. NeuroImage, 61(2), 450-456. doi:10.1016/j.neuroimage.2011.12.040.

Posner, M. I., \& Rothbart, M. K. (2007). Research on attention networks as a model for the integration of psychological science. Annual Review of Psychology, 58, 1-23. doi:10.1146/annurev.psych.58. 110405.085516.

Raffone, A., \& Srinivasan, N. (2017). Mindfulness and cognitive functions: toward a unifying neurocognitive framework. Mindfulness, 8(1), 1-9. doi:10.1007/s12671-016-0654-1.

Reijnders, J., van Heugten, C., \& van Boxtel, M. (2013). Cognitive interventions in healthy older adults and people with mild cognitive impairment: a systematic review. Ageing Research Reviews, 12(1), 263-275. doi:10.1016/j.arr.2012.07.003.

Reuter-Lorenz, P. (2002). New visions of the aging mind and brain. Trends in Cognitive Sciences, 6(9), 394.

Salthouse, T. A. (2004). What and when of cognitive aging. Current Directions in Psychological Science, 13(4), 140-144. doi:10.1111/ j.0963-7214.2004.00293.x.

Scarmeas, N., \& Stern, Y. (2003). Cognitive reserve and lifestyle. Journal of Clinical and Experimental Neuropsychology, 25(5), 625-633.

Seeley, W. W., Menon, V., Schatzberg, A. F., Keller, J., Glover, G. H., Kenna, H., et al. (2007). Dissociable intrinsic connectivity networks for salience processing and executive control. Journal of Neuroscience, 27(9), 2349-2356. doi:10.1523/JNEUROSCI.558706.2007.

Shapiro, S. L., Carlson, L. E., Astin, J. A., \& Freedman, B. (2006). Mechanisms of mindfulness. Journal of Clinical Psychology, 62(3), 373-386. doi:10.1002/jclp.20237.

Simen, A. A., Bordner, K. A., Martin, M. P., Moy, L. A., \& Barry, L. C. (2011). Cognitive dysfunction with aging and the role of inflammation. Therapeutic advances in chronic disease, 2(3), 175-195. doi: 10.1177/2040622311399145.

Slagter, H. A., Davidson, R. J., \& Lutz, A. (2011). Mental training as a tool in the neuroscientific study of brain and cognitive plasticity. Frontiers in Human Neuroscience, 5, 17. doi:10.3389/fnhum. 2011.00017.

Steffener, J., Brickman, A. M., Rakitin, B. C., Gazes, Y., \& Stern, Y. (2009). The impact of age-related changes on working memory functional activity. Brain Imaging and Behavior, 3(2), 142-153. doi:10.1007/s11682-008-9056-x.

Steffener, J., \& Stern, Y. (2012). Exploring the neural basis of cognitive reserve in aging. Biochimica et Biophysica Acta, 1822(3), 467-473. doi:10.1016/j.bbadis.2011.09.012.
Stern, Y. (2002). What is cognitive reserve? Theory and research application of the reserve concept. Journal of the International Neuropsychological Society, 8(3), 448-460.

Stern, Y. (2003). The concept of cognitive reserve: a catalyst for research. Journal of Clinical and Experimental Neuropsychology, 25(5), 589593.

Stern, Y. (2009). Cognitive reserve. Neuropsychologia, 47(10), 20152028. doi:10.1016/j.neuropsychologia.2009.03.004.

Tabert, M. H., Albert, S. M., Borukhova-Milov, L., Camacho, Y., Pelton, G., Liu, X., et al. (2002). Functional deficits in patients with mild cognitive impairment: prediction of AD. Neurology, 58(5), 758-764.

Tang, Y. Y., Ma, Y., Fan, Y., Feng, H., Wang, J., Feng, S., et al. (2009). Central and autonomic nervous system interaction is altered by short-term meditation. Proceedings of the National Academy of Sciences, 106(22), 8865-8870.

Tang, Y. Y., Ma, Y., Wang, J., Fan, Y., Feng, S., Lu, Q., et al. (2007). Short-term meditation training improves attention and self-regulation. Proceedings of the National Academy of Sciences of the USA, 104(43), 17152-17156. doi:10.1073/pnas.0707678104.

Tang, Y. Y., \& Posner, M. I. (2009). Attention training and attention state training. Trends in Cognitive Sciences, 13(5), 222-227. doi:10. 1016/j.tics.2009.01.009.

Tang, Y. Y., \& Posner, M. I. (2014). Training brain networks and states. Trends in Cognitive Sciences, 18(7), 345-350. doi:10.1016/j.tics. 2014.04.002.

Tang, Y. Y., Rothbart, M. K., \& Posner, M. I. (2012). Neural correlates of establishing, maintaining, and switching brain states. Trends in Cognitive Sciences, 16(6), 330-337. doi:10.1016/j.tics.2012.05.001.

Taylor, V. A., Daneault, V., Grant, J., Scavone, G., Breton, E., RoffeVidal, S., et al. (2013). Impact of meditation training on the default mode network during a restful state. Social Cognitive and Affective Neuroscience, 8(1), 4-14. doi:10.1093/scan/nsr087.

Ulrich-Lai, Y. M., \& Herman, J. P. (2009). Neural regulation of endocrine and autonomic stress responses. Nature Reviews Neuroscience, 10(6), 397-409. doi:10.1038/nrn2647.

van Leeuwen, S., Müller, N. G., \& Melloni, L. (2009). Age effects on attentional blink performance in meditation. Consciousness and Cognition, 18(3), 593-599. doi:10.1016/j.concog.2009.05.001.

Veehof, M. M., Oskam, M. J., Schreurs, K. M., \& Bohlmeijer, E. T. (2011). Acceptance-based interventions for the treatment of chronic pain: a systematic review and meta-analysis. Pain, 152(3), 533-542. doi:10.1016/j.pain.2010.11.002.

Wallace, B. A. (1999). The Buddhist tradition of samatha: methods for refining and examining consciousness. Journal of Consciousness Studies, 6(2-3), 175-187.

Wallace, B. A. (2005). Balancing the mind: a Tibetan buddhist approach to refining attention. Ithaca: Snow Lion.

Wangchug Dorje, K. (2009). Mahamudra: ocean of true meaning. Münster: Edition Octopus.

Wells, R. E., Yeh, G. Y., Kerr, C. E., Wolkin, J., Davis, R. B., Tan, Y., et al. (2013). Meditation's impact on default mode network and hippocampus in mild cognitive impairment: a pilot study. Neuroscience Letters, 556, 15-19. doi:10.1016/j.neulet.2013.10.001.

Williams, J. M. G., Crane, C., Barnhofer, T., Brennan, K., Duggan, D. S., Fennell, M. J., et al. (2014). Mindfulness-based cognitive therapy for preventing relapse in recurrent depression: a randomized dismantling trial. Journal of Consulting and Clinical Psychology, 82(2), 275-286.

Xiong, G. L., \& Doraiswamy, P. M. (2009). Does meditation enhance cognition and brain plasticity? Annals of the New York Academy of Sciences, 1172, 63-69. doi:10.1196/annals.1393.002. 